\title{
An entertainment-education approach to prevent COVID-19 spread: study protocol for a multi-site randomized controlled trial
}

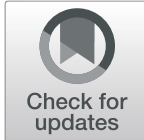

\author{
Alain Vandormael $^{1^{*}}$ (D), Maya Adam², Merlin Greuel ${ }^{1}$ and Till Bärnighausen ${ }^{1,3,4}$
}

\begin{abstract}
Background: Entertainment-education (E-E) media can improve behavioral intent toward health-related practices. In the era of COVID-19, millions of people can be reached by E-E media without requiring any physical contact. We have designed a short, wordless, animated video about preventive COVID-19 behaviors that can be rapidly distributed through social media channels to a global audience. The E-E video's effectiveness, however, remains unclear.

Methods/design: This is a multi-site, parallel group, randomized controlled trial comparing the effectiveness of an E-E video on COVID-19 against (i) an attention placebo control (APC) video and (ii) no video. For our primary outcomes, we will measure knowledge about preventive COVID-19 behaviors. We will also use a list randomization approach to measure behavioral intent toward preventative COVID-19 behaviors. In each trial arm, participants will be randomized to a control list or a control list plus an item about social distancing, washing hands, cleaning household surfaces, sharing of eating utensils, and the stockpiling of essential goods. Using an online platform, we will recruit 17,010 participants (aged 18-59 years) from the USA, the UK, Germany, Spain, France, and Mexico.

Trial registration: German Clinical Trials Register \#DRKS00021582. Registered on May 12, 2020.

Discussion: This trial will utilize several randomization procedures, list experimentation methods, and state-of-theart online technology to demonstrate the effectiveness of an E-E video to improve knowledge of, and behavioral intent toward, the prevention of COVID-19. Our results will inform future E-E video campaigns for COVID-19 and similar public health intervention needs.
\end{abstract}

Keywords: COVID-19, Randomized controlled trial, Protocol, Entertainment-education, Behavioral intent, Knowledge, List experiment

\footnotetext{
* Correspondence: alain.vandormael@uni-heidelberg.de

${ }^{1}$ Heidelberg Institute of Global Health, University of Heidelberg, Heidelberg, Germany

Full list of author information is available at the end of the article
}

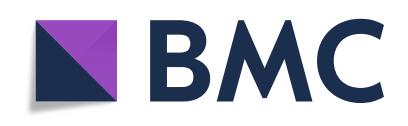

(- The Author(s). 2020 Open Access This article is licensed under a Creative Commons Attribution 4.0 International License, which permits use, sharing, adaptation, distribution and reproduction in any medium or format, as long as you give appropriate credit to the original author(s) and the source, provide a link to the Creative Commons licence, and indicate if changes were made. The images or other third party material in this article are included in the article's Creative Commons licence, unless indicated otherwise in a credit line to the material. If material is not included in the article's Creative Commons licence and your intended use is not permitted by statutory regulation or exceeds the permitted use, you will need to obtain permission directly from the copyright holder. To view a copy of this licence, visit http://creativecommons.org/licenses/by/4.0/. The Creative Commons Public Domain Dedication waiver (http://creativecommons.org/publicdomain/zero/1.0/) applies to the data made available in this article, unless otherwise stated in a credit line to the data. 


\section{Administrative information}

\begin{tabular}{|c|c|}
\hline Title & $\begin{array}{l}\text { An entertainment-education approach } \\
\text { to prevent COVID-19 spread: study } \\
\text { protocol for a multi-site randomized } \\
\text { controlled trial }\end{array}$ \\
\hline Trial registration & $\begin{array}{l}\text { The study and its outcomes were } \\
\text { registered at the German Clinical Trials } \\
\text { Register (www.drks.de) on May 12th, } \\
\text { 2020: \#DRKS00021582. }\end{array}$ \\
\hline Protocol version & 1.1, 4 June 2020. \\
\hline Funding & $\begin{array}{l}\text { This study is funded by an Alexander } \\
\text { von Humboldt University Professor Prize } \\
\text { awarded to Dr. Till Bärnighausen. }\end{array}$ \\
\hline Author details & $\begin{array}{l}1 \text { Heidelberg Institute of Global Health, } \\
\text { University of Heidelberg, Heidelberg, } \\
\text { Germany } \\
2 \text { Department of Pediatrics, Stanford } \\
\text { University School of Medicine, Stanford, } \\
\text { CA, USA } \\
3 \text { Department of Global Health and } \\
\text { Population, Harvard T. H Chan School } \\
\text { of Public Health, Boston, USA. } \\
4 \text { Africa Health Research Institute (AHRI), } \\
\text { Somkhele, KwaZulu-Natal, South Africa }\end{array}$ \\
\hline $\begin{array}{l}\text { Name and contact } \\
\text { information for the trial } \\
\text { sponsor }\end{array}$ & Not applicable \\
\hline Role of sponsor & Not applicable \\
\hline
\end{tabular}

\section{Background and rationale}

A large amount of information about novel coronavirus (COVID-19) has been disseminated by the traditional mass media since the outbreak of the pandemic [1-3]. However, it is not clear if this dissemination has improved knowledge of, or intent toward, preventive COVID-19 behaviors. By preventive behaviors, we mean the public's adoption of practices, such as social distancing, reduced physical contact, and hand/surface sanitization (among others), to reduce the spread of COVID-19.

Arguably, one possible limitation of mainstream mass media is that it has been perceived as politicized and culturally localized, thus limiting the persuasiveness of messages about COVID-19 prevention [4, 5]. It is also likely that traditional mass media channels (e.g., local or national television networks) have missed key segments of the population, such as young people (25-40 years) who are disproportionately more likely to transmit the virus to older people ( $\geq 50$ years) [6]. An effective public health response could therefore benefit from entertainment-education (E-E) approaches that increase COVID-19 prevention outcomes [7].

To improve knowledge and behavioral intent toward COVID-19 prevention, we have designed an animated, wordless, E-E video that can be rapidly distributed to a diverse and global audience through social media channels. With a short duration (approximately 2.30 min), the E-E video contains no speech and minimizes cultural signifiers to increase universality and appeal. The E-E video was released on Stanford Medicine's YouTube channel on March 21, 2020, and went viral within $24 \mathrm{~h}$. After 10 days, it had reached 332,000 views on YouTube, 220,000 views on Instagram, 294,000 views on Facebook, and 402,000 views on Twitter, with a cumulative count of 1.2 million views [8]. The E-E video could play a useful role in disseminating evidence-based health recommendations related to COVID-19; however, its effectiveness remains unclear.

\section{Objectives}

The study aims to achieve the following objectives. To establish:

1. The E-E video's effectiveness in improving knowledge of preventive COVID-19 behaviors

2. The E-E video's effectiveness in increasing behavioral intent toward COVID-19 prevention

3. People's voluntary interest to watch the E-E video

\section{Trial design}

The present study is a multi-site, parallel group, randomized controlled trial (RCT) comparing the effectiveness of the E-E video against an APC video or no video (Fig. 1) [9]. Randomization is at a 1:1:1 ratio for the three arms of the trial. Nested within each arm of the trial is a list experiment. Participants will be randomized at a 1:1 ratio to a control or treatment list. After completing the list experiment, participants in arms 2 and 3 will receive the E-E video to ensure post-trial access to treatment [10].

\section{Methods: participants, interventions, and outcomes \\ Study setting}

This will be an online study setting. We will use the online recruitment platform Prolific Academic (ProA: https://www.prolific.co) to enroll participants from the USA, the UK, Germany, Spain, Mexico, and France. We will host and deploy our study on an online platform called Gorilla $^{\mathrm{mw}}$ (www.gorilla.sc) (Fig. 2).

\section{Eligibility criteria}

Registered participants on the ProA platform must be between the ages of 18 and 59 years (male, female, or other), have current residence in the target countries, and speak English, German, or Spanish.

\section{Ethical approval}

Ethical approval was obtained from the Stanford University IRB on April 12, 2020, protocol \#55820. 


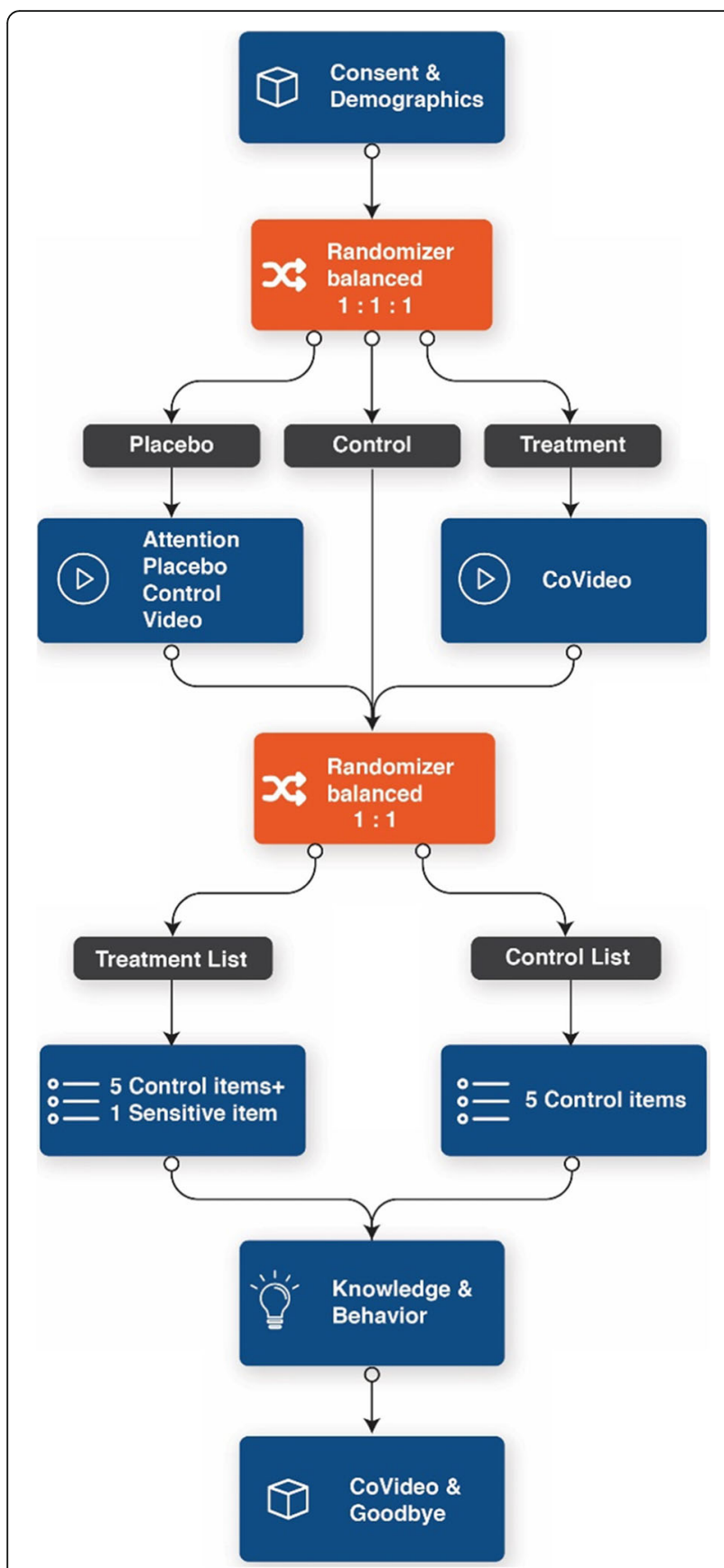

Fig. 1 The trial design, which consists of three arms. Participants will be randomly assigned (at a 1:1:1 ratio) to the E-E video (CoVideo, arm 1), an APC video (arm 2), or no video (arm 3). Each arm has a list experiment, with participants split (at a 1:1 ratio) into a control or treatment group

\section{Who will take informed consent?}

Participants will undergo a process of informed consent on the ProA platform. The consent form explains the purpose of the study, the risks and benefits of the research, and how to contact the study investigators (or the Stanford University ethics review board) about problems experienced during the course of the study.

\section{Criteria for discontinuing or modifying allocated interventions}

We will not discontinue or modify the allocated interventions during the course of the study. Participants may choose to discontinue their participation at any time.

\section{Interventions \\ Intervention description}

The intervention is an E-E video about COVID-19 prevention, which we abbreviate to CoVideo. Developed by our co-author (MA) for Stanford Medicine, the CoVideo is animated with sound effects and has no words, speech, or text. The CoVideo shows how the novel coronavirus is spread (airborne, physical contact) and recommends best practices to prevent onward transmission (staying at home, not congregating in public spaces, and sanitizing hands/surfaces). The CoVideo also covers the mass media coverage of the outbreak and the public's response to this media coverage, which includes a subplot on the stockpiling of essential goods, and the impact thereof on healthcare services and resources (e.g., doctors being unable to access protective equipment). The CoVideo can be viewed at https://www.youtube.com/ watch? $v=r A j 38 E 7 v r S 8$.

\section{Explanation for the choice of comparators}

The comparators are an APC video (arm 2) or no video (arm 3). The APC video is similar in style to the CoVideo; it is also animated with a duration of $2.30 \mathrm{~min}$ and has sound effects but no words, speech, or text. The video message is about how small choices become actions, which become habits, which become a way of life (https://www.youtube.com/watch?v=_HEnohs6yYw). The APC will enable us to quantify the attention effect of the CoVideo. APC conditions should mimic the "inactive" components of an intervention-the effect of watching the video-while not containing any of the "active" intervention components-the content delivered by the video [11]. To measure its total effect, we also compare the CoVideo with a control (no video). We do not assume the CoVideo is better than nothing; it is possible that the CoVideo could motivate reactance to our message about COVID-19 prevention [12-14]. For the list experiments, we will use the control list as the comparator.

\section{Outcomes}

\section{Primary outcome measures}

There are two primary outcome measures. First, we will use 18 True/False questions to assess if the CoVideo 


\begin{tabular}{|c|c|c|c|}
\hline & \multicolumn{3}{|c|}{ STUDY PERIOD } \\
\hline & Enrolment & Allocation & $\begin{array}{c}\text { Post- } \\
\text { allocation }\end{array}$ \\
\hline TIMEPOINT & $\begin{array}{c}-t_{0} \\
\text { Minute } 1\end{array}$ & $\begin{array}{c}t_{0} \\
\text { Minute } 0\end{array}$ & $\begin{array}{c}t_{1} \\
\text { Minute 1-10 }\end{array}$ \\
\hline \multicolumn{4}{|l|}{ ENROLMENT: } \\
\hline \multirow{2}{*}{$\begin{array}{l}\text { Eligibility screen } \\
\text { Informed consent }\end{array}$} & $X$ & & \\
\hline & $x$ & & \\
\hline Allocation & & $X$ & \\
\hline INTERVENTIONS: & & & $x$ \\
\hline \multirow{2}{*}{$\begin{array}{l}\text { Arm } 1 \\
\text { Arm } 2\end{array}$} & & & $\mathrm{x}$ \\
\hline & & & $X$ \\
\hline Arm 3 & & & $X$ \\
\hline \multirow{2}{*}{$\begin{array}{r}\text { ASSESSMENTS: } \\
\text { Questionnaire } \\
\text { survey }\end{array}$} & & & $x$ \\
\hline & & & $X$ \\
\hline
\end{tabular}

Fig. 2 Schedule of enrolment, interventions, and assessments for the study

improves knowledge about preventive COVID-19 behaviors. These questions are shown in Table 1 and this data will be used to assess objective 1 .

Second, we will assess if the CoVideo improves behavioral intent toward preventive COVID-19 behaviors. Since many participants will be primed to give socially desirable answers to the behavioral intent questions, we will use a list randomization approach [15-17]. There will be five list experiments, which are shown in Table 2. For each experiment, the control group will receive a list of five items. Participants will be asked to state how many of the items they agree with, without stating which ones they agree with. For the first list experiment, imagine that the control group selects an average of 2 out of the 5 items. The treatment group will get the same list but with one additional "sensitive" item about behavioral intent. Using the example, the participants in the treatment group select an average of 2.2 out of the 6 items. Holding all else equal, we conclude that the prevalence of participants that would go out with friends (item 6 of list 1 in Table 2), in defiance of lockdown regulations, is $20 \%$. We designed the list experiments in line with best practices [17], and we will use this data to assess objective 2 .

\section{Secondary outcome measure}

We will assess participant's behavioral intent to seek health information from online, animated videos. Because of social desirability bias, we will again use a list experiment with the same procedures described in Table 2. The list experiment will read: This week, I will seek health information from: Television news channels; Social media celebrities; Religious leaders on the Internet; Public health agency websites; Conversations with family and friends; "Animated videos made by health experts. (The sensitive item is indicated by an asterisk (*).)

We aim to measure participant engagement with the CoVideo. At the end of the survey, we will offer the $\mathrm{APC}$ and no-video arms the choice to watch the CoVideo or end the survey. The Gorilla platform will record this response. If the "Watch Video" button is clicked, Gorilla will record the time (in milliseconds) from the video start until the participant clicks the "Finish" button (or until the video ends at $2.30 \mathrm{~min}$, whichever comes first). This data will be used to assess objective 3 . 
Table 1 The COVID-19 knowledge items, which require True/False responses

\begin{tabular}{|c|c|}
\hline $\begin{array}{l}\text { The current coronavirus can be spread by an infected person } \\
\text { even if they look healthy }\end{array}$ & $\begin{array}{l}\text { Cleaning surfaces with soap and water is an effective way to kill the current } \\
\text { coronavirus }\end{array}$ \\
\hline $\begin{array}{l}\text { The current coronavirus cannot be spread from person to } \\
\text { person }\end{array}$ & $\begin{array}{l}\text { An effective way to prevent COVID-19 spread is to wear a face mask even if you do } \\
\text { not have COVID-19 symptoms }\end{array}$ \\
\hline $\begin{array}{l}\text { The current coronavirus cannot survive on surfaces for more } \\
\text { than a few minutes }\end{array}$ & $\begin{array}{l}\text { An effective way to prevent COVID-19 spread is to wash your hands frequently with } \\
\text { soap and water }\end{array}$ \\
\hline $\begin{array}{l}\text { Some people with COVID-19 infection may experience a } \\
\text { cough }\end{array}$ & $\begin{array}{l}\text { An effective way to prevent COVID-19 spread is to regularly rinse your nose with salt } \\
\text { water }\end{array}$ \\
\hline $\begin{array}{l}\text { Some people with COVID-19 infection do not experience a } \\
\text { fever }\end{array}$ & An effective way to prevent COVID-19 spread is to avoid touching your face \\
\hline $\begin{array}{l}\text { The current coronavirus spreads from person to person } \\
\text { through small droplets from the mouth }\end{array}$ & $\begin{array}{l}\text { An effective way to prevent COVID-19 spread is to avoid shaking hands with other } \\
\text { people }\end{array}$ \\
\hline $\begin{array}{l}\text { The current coronavirus spreads from person to person } \\
\text { through small droplets from the nose }\end{array}$ & $\begin{array}{l}\text { An effective way to prevent COVID-19 spread is to avoid places that are crowded with } \\
\text { people (like bars, restaurants, or performances) }\end{array}$ \\
\hline $\begin{array}{l}\text { You can catch COVID-19 by touching a contaminated surface } \\
\text { and then touching your face }\end{array}$ & An effective way to prevent COVID-19 spread is to eat garlic with each meal \\
\hline Antibiotics can be used to treat COVID-19 infection & $\begin{array}{l}\text { An effective way to prevent COVID-19 spread is to avoid sharing eating utensils with } \\
\text { others }\end{array}$ \\
\hline
\end{tabular}

\section{Sample size}

We calculated the sample size needed for pairwise comparisons between three groups using a one-way analysis of variance (ANOVA). The formula to calculate the sample size is [18]:

$$
n_{A}=\left(\sigma_{A}^{2}+\sigma_{B}^{2} / \kappa\right)\left(\frac{Z_{1-\frac{\alpha}{\tau}}+Z_{1-\beta}}{\mu_{A}-\mu_{B}}\right)^{2}
$$

where $\kappa=1$, which is the matching ratio; $\mu_{A}$ and $\mu_{B}$ are the group A and B means; $\sigma_{A}$ and $\sigma_{B}$ are the group A and $\mathrm{B}$ standard deviations; $\alpha=0.05$ is the type-I error; $\beta=0.20$ is the type-II error; $Z$ is the quantile function; and $\tau=2$ is the number of comparisons to be made. To detect a small difference of 0.1 between the knowledge scores of two trial arms, we assumed a mean of $\mu_{A}=$ 14.0 and $\mu_{B}=14.1$ and $\sigma_{A}=1.5$ and $\sigma_{B}=1.5$. We select $\mu$ on the expectation that participants will get 14 out of 18 knowledge items correct. This gives a sample size of $n_{A}=n_{B}=n_{C}=3,532$, so $N=10,596$. If we change our assumption about $\sigma$ and increase it to $\sigma=1.8$, then the required sample size becomes $n_{A}=n_{B}=n_{C}=5,087$ and $N=15,261$. For the list experiment, we selected a sample size to detect a small difference of 0.05 between the control list group and the treatment list group. Because we framed the list questions to avoid floor and ceiling effects, we expect, on average, that the control group will agree with 2 out of the 5 items and the treatment group 2.05 of the 6 items. We selected $\sigma_{A}=0.7$ and $\sigma_{B}=0.8$, allowing the second $\sigma$ to be larger because of the additional sensitive item. This calculation gives a sample size of $n_{A}=n_{B}=n_{C}=3,548$ and $N=10,644$. If we change our assumption about $\sigma$ and increase it to $\sigma_{A}=0.9$ and
$\sigma_{B}=1.0$, then we have $n_{A}=n_{B}=n_{C}=4,553$ and $N=$ 13,659 . For this study, we will recruit 17,010 participants, more than the 15,261 calculated sample size, to ensure our study is sufficiently powered.

\section{Recruitment}

To be recruited, a person must open an account on ProA and provide his or her personal information. Participants must agree to ProA's data privacy terms and conditions. ProA will assign each participant a unique, anonymized ID. The study investigators will also open an account on ProA. We will instruct the ProA platform on how many participants need to be recruited. ProA will filter out all participants who do not meet the eligibility criteria. Participant recruitment will happen on a "first come, first served" basis until the recruitment number (sample size) is reached. We will compensate the participants an equivalent of $£ 1$ for the expected 10min completion time.

\section{Assignment of interventions: allocation}

The Gorilla platform is designed to host and implement online experimental studies (implementation). Gorilla will randomly allocate participants to the intervention (CoVideo), placebo (APC), or control (no video) arm (sequence generation). Gorilla will use a web-based randomization algorithm, which is unknown to us (concealment mechanism).

\section{Assignment of interventions: blinding Who will be blinded}

The study investigators and those involved in the data analyses and statistics will be blinded to the group allocation. 
Table 2 In each trial arm, both groups will receive five lists. For each list, the control group will get the first five items only; the treatment group will receive the five items and the sixth sensitive item, indicated by an asterisk $\left({ }^{*}\right)$. Each list experiment will be preceded by the question: "How many of the five/six statements do you agree with? We don't want to know which ones, just answer how many. This week I will spend time watching TV, etc."

\section{List 1: Social distancing \\ 1. Spend time watching TV \\ 2. Do the vacuuming \\ 3. Pick a fight with my partner \\ 4. Eat a low sugar diet \\ 5. Rinse my nose with salt water daily \\ 6. Go out with my friends*}

\section{List 3: Clean surfaces}

1. Watch a new TV series

2. Spend time gardening by myself

3. Try to go vegetarian

4. Have alcoholic drinks on at least three evenings

5. Catch up on last week's work

6. Clean kitchen counters after use $e^{*}$

\section{List 5: Stockpiling}

1. Spend time chatting with my friends online

2. Try new cooking recipes

3. Watch a pornographic movie

4. Clean all floor surfaces

5. Visit the World Health Organization (WHO) website

6. Stock up on household supplies for a month*

\section{Data collection and management}

Plans for assessment and collection of outcomes

Data will be collected on the Gorilla platform. Participants will submit data by clicking on the response buttons. We expect to collect the data over a 4-week period.

\section{Plans to promote participant retention and complete follow-up}

Participants will automatically exit the study if they take longer than $45 \mathrm{~min}$ to complete the survey. The timeout is to ensure that participants do not clog up the system with incomplete surveys. Since the participants are anonymous to us, there is no way to initiate follow-up in the time limit.

\section{Data management}

Gorilla will store the trial data on its cloud platform, hosted on Microsoft Azure in the Republic of Ireland. The Gorilla database is encrypted using industrystandard cryptography. The study investigators own the research data that has been collected using Gorilla and have complete control over it. The study investigators can generate and access the completely anonymized data from the Gorilla platform. The data will be downloaded and safely stored for statistical analysis on a computing system maintained by the University of Heidelberg in Germany.

\section{Confidentiality}

The participants, who are completely anonymous to us, will have no identifying information associated with their unique IDs. We will inform participants that if they email the study investigators then their names could be revealed to us. The study investigators will keep this information confidential.

\section{Statistical methods \\ Descriptive measures}

We will use descriptive statistics to obtain the mean and standard deviation of age, the proportion of participants by gender, and frequency distributions for country of residence and education status.

\section{Primary outcomes}

For each participant, we will sum the knowledge items that are correctly answered $(\min .=0, \max .=18)$. We will then obtain the mean knowledge score for each trial arm. Let $\bar{K}_{k}$ denote the mean knowledge score for the $k$ th trial arm, where $k \in\{v, a, c\}$ such that $v$ represents the CoVideo, $a$ represents the APC, and $c$ represents the control. We will use an ordinary least squares (OLS) regression model to estimate $\bar{K}_{k}$ as well as obtain the difference estimators. We define the difference between the CoVideo $\left(\bar{K}_{v}\right)$ and the control $\left(\bar{K}_{c}\right)$ as the total effect, the difference between the CoVideo $\left(\bar{K}_{v}\right)$ and the $\operatorname{APC}\left(\bar{K}_{a}\right)$ as the content effect, and the difference between the APC video $\left(\bar{K}_{a}\right)$ and the control $\left(\bar{K}_{c}\right)$ as the attention effect.

For each list experiment, we will calculate the mean score for the control list, denoted by $\overline{C_{i}}$, and the mean score for the treatment list, denoted by $\overline{T_{i}}$, for the $i$ th list experiment $(i=1, \ldots, 5)$. We will then estimate the difference between the treatment and control list for each trial arm, denoted by $D_{i k}=\bar{T}_{i k}-\bar{C}_{i k}$, for the $i$ th treatment list and $k$ th trial arm, where $k \in\{v, a, c\}$. Let $D$ 
$D_{i}^{T}$ denote the total effect, which is estimated by $D_{i v}-$ $D_{i c}$; let $D D_{i}^{C}$ denote the content effect, which is estimated by $D_{i v}-D_{i a}$; and let $D D_{i}^{A}$ denote the attention effect, which is estimated by $D_{i a}-D_{i c}$. These analyses are analogous to difference-in-difference analyses, which can be implemented by specifying the appropriate main and interaction terms in an OLS regression.

\section{Secondary outcomes}

Using the same statistical procedure described above, we will assess behavioral intent to seek health information with E-E media. To establish participant engagement with the CoVideo, we will use a logistic regression model to estimate the proportion of participants in the APC and control arms that chose to watch the CoVideo, adjusting for the knowledge scores and demographic factors. We will use either an OLS model or Cox proportional hazards model to model the time to when a person clicks "Finish Video" (right censoring) or when the CoVideo ends naturally (left censoring). We will use $\mathrm{R}$ statistical software to undertake the analysis.

\section{Interim analyses}

No interim analyses are planned.

\section{Methods for additional analyses (e.g., subgroup analyses)} We will conduct both country-specific and crosscountry pooled analyses, provided sufficient sample sizes can be recruited from each country.

\section{Methods in analysis to handle protocol non-adherence and any statistical methods to handle missing data}

Participants will have a 30-s time limit to answer each knowledge item. This limit is to prevent participants from searching for answers on the Internet. If the participant times out, they will receive a missing value of 9 . This missing value will be recoded as an incorrect answer to the knowledge item, since the participant could not correctly answer the question in the allotted time.

\section{Plans to give access to the full protocol, participant level- data, and statistical code}

This document is the full protocol. Anyone interested in other data or documentation should contact the corresponding author.

\section{Oversight and monitoring Composition of the coordinating center and trial steering committee}

The trial will be overseen by a trial steering committee (TSC). The TSC will have an independent chairperson and members but also includes the trial collaborators. Two TSC meetings are planned.

\section{Adverse event reporting and harms}

It is unlikely there will be adverse events given the online format of the trial. The auditing of trial conduct will be addressed in the two TSC meetings.

\section{Dissemination plans}

We will disseminate the study findings through journal publications and conference presentations.

\section{Discussion}

There is a critical need for public health actors to disseminate scientific information about the prevention of COVID-19, especially in the absence of a vaccine. To this end, we have produced a short, animated E-E video to improve knowledge of, and behavioral intent, toward preventive COVID-19 behaviors. In this study, we propose to evaluate the effectiveness of our E-E video using three innovative approaches.

First, we will use state-of-the-art online technology to implement our multi-site randomized controlled trial. The ProA platform will enable us to rapidly recruit a large and diverse number of participants who speak different languages and live in different countries in Europe, North America, and Latin America. Second, we will implement the study on the Gorilla platform, which is designed to host and facilitate experimental trials in behavioral research. The Gorilla platform will enable us to randomize at two different levels: (1) participants will be randomized to the $\mathrm{E}-\mathrm{E}$ intervention, the $\mathrm{APC}$, or the control arm; (2) within each arm, participants will then be randomized to receive a control list or treatment list. Further, the list experiments will also be randomly ordered to avoid order effects. All randomization will be performed automatically by the Gorilla randomization algorithm, which demonstrates the platform's potential to implement innovative trial designs and concepts.

Third, we will use a list experiment as the primary outcome of this study. Given the unprecedented nature of the COVID-19 outbreak, and the media attention it has received, we assume that participants will already be primed to give socially acceptable responses to questions about COVID-19. In public, individuals will say they will adhere to social distancing recommendations. But in private, they may attend secret "corona parties" [19]. The list experiment gets around the problem of eliciting untruthful answers to socially desirable questions. Further, we have designed the trial in a way, making full use of the Gorilla features, to measure the magnitude of social desirability bias toward the COVID-19 questions.

We expect that our study will make important contributions to the E-E literature. Importantly, lessons learned can help us to improve the design of E-E videos to disseminate public health information for future pandemics. And we may hopefully demonstrate that our E-E 
video, which has been viewed more than a million times on social media thus far, will have contributed to current public health efforts to keep people safe and reduce the spread of COVID-19. Our results will also guide future E-E strategies to support the long-term COVID-19 response, as countries are easing first-wave lockdown or reimplementing second-wave lockdowns.

\section{Trial status}

The trial is currently recruiting on the ProA website. The first 50 participants were randomized to the pilot phase of the trial on March 23, 2020. A structured summary of the protocol was published on June 3, 2020 [9]. Full recruitment began on June 5, 2020. Thus far, 14,800 surveys have been completed. Recruitment is due to end June 25, 2020. The trial was registered on the German Clinical Trials Register (www.drks.de) on May 12, 2020, with registration number \#DRKS00021582.

\section{Acknowledgements}

Not applicable.

\section{Authors' contributions}

AV wrote the protocol. MA, MG, and TB provided comments and feedback. MA designed the E-E video. TB and AV designed the trial. All authors contributed to the questionnaire development. The authors read and approved the final manuscript.

\section{Funding}

This study is funded by the Alexander von Humboldt University Professor Prize awarded to TB (last author). The funder played no role in the design of the study and collection, analysis, and interpretation of data and in writing the manuscript. Open Access funding enabled and organized by Projekt DEAL.

\section{Availability of data and materials}

Data will be collected and stored on the Gorilla platform. The study investigators own and have complete control of the research data, which can be accessed at any time. For statistical analysis, the data will be downloaded and safely stored on a computing system maintained by the University of Heidelberg.

\section{Consent for publication}

Not applicable.

\section{Competing interests}

The authors declare that they have no competing interests.

\section{Author details}

${ }^{1}$ Heidelberg Institute of Global Health, University of Heidelberg, Heidelberg, Germany. ${ }^{2}$ Department of Pediatrics, Stanford University School of Medicine, Stanford, CA, USA. ${ }^{3}$ Department of Global Health and Population, Harvard T. $\mathrm{H}$ Chan School of Public Health, Boston, USA. ${ }^{4}$ Africa Health Research Institute (AHRI), Somkhele, KwaZulu-Natal, South Africa.

Received: 18 June 2020 Accepted: 1 December 2020 Published online: 15 December 2020

\section{References}

1. Cinelli M, Quattrociocchi W, Galeazzi A, et al. The COVID-19 social media infodemic. arXiv. 2020; http://arxiv.org/abs/2003.05004 (Accessed March 31, 2020).

2. Mian A, Khan S. Coronavirus: the spread of misinformation. BMC Med. 2020;18:89.

3. Kouzy R, Abi Jaoude J, Kraitem A, et al. Coronavirus goes viral: quantifying the COVID-19 misinformation epidemic on Twitter. Cureus. 2020. https://doi. org/10.7759/cureus.7255.
4. Garfin DR, Silver RC, Holman EA. The novel coronavirus (COVID-2019) outbreak: amplification of public health consequences by media exposure. Health Psychol. 2020; In Press. https://doi.org/10.1037/hea0000875.

5. Garrett L. COVID-19: the medium is the message. Lancet. 2020;395:942-3.

6. Zhao Z, Zhu Y-Z, Xu J-W, et al. A mathematical model for estimating the agespecific transmissibility of a novel coronavirus. medRxiv. 2020; 2020.03.05. 20031849. https://www.medrxiv.org/content/10.1101/2020.03.05.20031849v1.

7. Hahn U, Lagnado D, Lewandowsky S, Chater N. Crisis knowledge management: reconfiguring the behavioural science community for rapid responding in the Covid-19 crisis. psyarxiv. 2020. https://psyarxiv.com/hsxdk/.

8. Adam M, Bärnighausen T, McMahon SA. Design for extreme scalability: a wordless, globally scalable COVID-19 prevention animation for rapid public health communication. J Glob Health. 2020;10:10343.

9. Vandormael A, Adam M, Greuel M, Bärnighausen T. A short, animated video to improve good COVID-19 hygiene practices: a structured summary of a study protocol for a randomized controlled trial. Trials. 2020;21:469.

10. Doval D, Shirali R, Sinha R. Post-trial access to treatment for patients participating in clinical trials. Perspect Clin Res. 2015;6:82.

11. Freedland KE, Mohr DC, Davidson KW, Schwartz JE. Usual and unusual care: existing practice control groups in randomized controlled trials of behavioral interventions. Psychosom Med. 2011;73:323-35.

12. Dillard JP, Shen L. On the nature of reactance and its role in persuasive health communication. Commun Monogr. 2005;72:144-68.

13. Miller CH, Lane LT, Deatrick LM, Young AM, Potts KA. Psychological reactance and promotional health messages: the effects of controlling language, lexical concreteness, and the restoration of freedom. Hum Commun Res. 2007;33:219-40.

14. Richards AS, Banas JA. Inoculating against reactance to persuasive health messages. Health Commun. 2015;30:451-60.

15. Kramon E, Weghorst K. (Mis) Measuring sensitive attitudes with the list experiment. Public Opin Q. 2019;83:236-63.

16. Corstange D. Sensitive questions, truthful answers? Modeling the list experiment with LISTIT. Polit Anal. 2009:17:45-63.

17. Glynn AN. What can we learn with statistical truth serum? Public Opin Q. 2013:77:159-72

18. Rosner B. Fundamentals of biostatistics. 7th ed. Boston: Brooks/Cole; 2010.

19. Levine J. New Yorkers are throwing 'corona potlucks' and visiting 'speakeasies'. New York Post 2020. https://nypost.com/2020/03/28/new-yorkers-arethrowing-corona-potlucks-and-visiting-speakeasies/. Accessed 14 Apr 2020.

\section{Publisher's Note}

Springer Nature remains neutral with regard to jurisdictional claims in published maps and institutional affiliations.
Ready to submit your research? Choose BMC and benefit from:
- fast, convenient online submission
- thorough peer review by experienced researchers in your field
- rapid publication on acceptance
- support for research data, including large and complex data types
- gold Open Access which fosters wider collaboration and increased citations
- maximum visibility for your research: over $100 \mathrm{M}$ website views per year
At BMC, research is always in progress.
Learn more biomedcentral.com/submissions 